Short communication

\title{
Experimental study of pullout capacity of plate anchors shallowly embedded in hydrate bearing sediments
}

\author{
Fang Liu ${ }^{\mathrm{a}, \mathrm{b}, *}$, Haoyu Sun ${ }^{\mathrm{c}}$, Jongwon Jung ${ }^{\mathrm{d}}$, Xuhui Zhang ${ }^{\mathrm{e}}$, Xin $\mathrm{Ju}^{\mathrm{c}}$ \\ ${ }^{\text {a }}$ State Key Laboratory of Disaster Reduction in Civil Engineering, Tongji University, China \\ ${ }^{\mathrm{b}}$ Key Laboratory of Geotechnical and Underground Engineering (Tongji University), Ministry of Education, China \\ ${ }^{\mathrm{c}}$ Department of Geotechnical Engineering, Tongii University, China \\ ${ }^{\mathrm{d}}$ School of Civil Engineering, Chungbuk National University, South Korea \\ ${ }^{\mathrm{e}}$ Institute of Mechanics, Chinese Academy of Sciences, China
}

\section{A R T I C L E I N F O}

\section{Keywords:}

Pullout capacity

Plate anchor

Gas hydrate

Physical model test

Floating offshore platform

\begin{abstract}
A B S T R A C T
Plate anchors are commonly used in mooring systems of offshore facilities for gas and oil production in deep and ultra-deep water. They may encounter hydrate-bearing sediments widespread in marine continental margins. The pullout capacity of square anchors embedded shallowly in a hydrate-bearing sand is investigated with smallscale model tests using tetrahydrofuran (THF) hydrate as the substitute of gas hydrate. The results show that the pullout capacity of the anchors increases with the increase of the anchor width and the burial depth. The pullout capacity of the anchors can be estimated by a dimensionless breakout factor. This factor increases with the increase of the burial depth ratio (defined as the ratio of the burial depth to the width of a plate anchor), exhibiting a trend similar to that obtained numerically and experimentally from plate anchors shallowly-embedded in undrained clays. Hydrate dissociation brings detrimental effects on the pullout capacity of the anchors. The pullout capacity of the anchors drops to $0.3 \%$ of the initial value after the hydrates dissociate. This pronounced reduction highlights the need for further investigation on the effect of oceanic hydrate dissociation on engineering performance of offshore facilities as a result of natural or anthropologic perturbation.
\end{abstract}

\section{Introduction}

As the oil and gas industry increasingly produces from deep water and hash environments due to world's escalating demands for hydrocarbons, offshore facilities face two geotechnical engineering challenges. First, more elaborate floating offshore platforms require innovative and economic anchoring foundation systems that transmit immense pullout forces to the ocean bed. Among the numerous anchoring systems, such as suction caissons, suction embedded or dragged in plate anchors, and dynamically embedded torpedo anchors (Randolph et al., 2011), plate-type anchors continue to be widely used because of their high capacity-to-weight ratios. The second challenge is that these anchoring systems must work under a wide range of deepwater soil conditions, such as soft fine-grained sediments (either clay, or in some regions carbonate muds and silts) and methane hydrate (MH) bearing sediments.

MHs are combustible crystalline compounds encaging methane molecules inside a water molecule lattice. They attract global research interests as a potential energy source for the massive quantities of reserves (Moridis et al., 2011). MHs are rather widespread in marine sediments where the temperature and pressure enable stable presence of hydrates. In particular, MHs are extensively found in outer continental margins with water depth more than $500 \mathrm{~m}$ (Kvenvolden and Lorenson, 2001), and thus deep water anchoring systems encountering $\mathrm{MH}$ oceanic reservoirs are expected to be common. This raises questions regarding engineering performance of anchoring systems in such $\mathrm{MH}$ environments. As suggested by many experimental studies, the presence of MHs significantly alters mechanical behaviours of the host sediments in terms of stiffness, strength and dilation, whilst the dissociation of MHs (as a result of salinity, temperature or/and pressure change arising from natural or anthropologic perturbations) could induce remarkable decay of strength in the host sediments (Kwon et al., 2008, 2010; 2013). Therefore, the complex properties of $\mathrm{MH}$ bearing sediments have considerable impacts on the pullout capacity of the anchoring systems that interact with MH-bearing sediments.

The pullout capacity of plate-type anchors has been investigated

\footnotetext{
* Corresponding author. State Key Laboratory of Disaster Reduction in Civil Engineering, Tongji University, China.

E-mail addresses: liufang@tongji.edu.cu (F. Liu), sunhaoyu@sunac.com.cn (H. Sun), jjung@chungbuk.ac.kr (J. Jung), zhangxuhui@imech.ac.cn (X. Zhang), juxin@tongji.edu.cn (X. Ju).
} 
with various approaches. In early studies, theoretical predictions were formulated through limit equilibrium analyses or limit analyses based on assumed or observed shapes of failure surfaces (Murray and Geddes, 1989; Rao and Kumar, 1994). These theories were later improved by using more sophisticated constitutive models for soils (Ardebili et al., 2016), and more rigorous numerical methods, for instance, the numerical limit-analysis (Merifield et al., 2006, 2001), the large-deformation finite element method (Wang et al., 2013, 2010) and the material point method (Coetzee et al., 2005). In addition, physical model tests performed either under natural gravity (1-g) or in centrifuges provides a means to study anchors under carefully controlled and monitored conditions. Centrifuge testing is capable of scaling the stresses in the model to represent full-scale conditions, but reproducing the stress-pressure-temperature conditions of $\mathrm{MHs}$ at seabed in a centrifuge environment is extremely difficult and often proves problematic (Zhang et al., 2015a). Alternatively, small-scale 1-g model testing is extensively used to calibrate and validate numerical models, thanks to simple equipment requirement, great flexibility and low cost (Chow et al., 2015; Das, 1995, 1978; Ilamparuthi et al., 2002; Ilamparuthi and Muthukrishnaiah, 1999). Even though the stress condition does not represent realistic seabed conditions, the scaling error can be reduced by presenting results in dimensionless forms and using model soils with constitutive responses similar to the prototype soils (Bradshaw et al., 2017). Most studies, regardless of the approaches employed, express the anchor's pullout capacity in terms of a dimensionless breakout factor. This factor increases with an increase in embedment depth until it becomes constant beyond a critical embedment depth, indicating a transition from "shallow anchor behaviour" to "deep anchor behaviour". In addition to the anchor embedment and geometry, various soil conditions have been examined, such as sands of various densities, and clays with uniform strength (Thorne et al., 2004) and linearly increasing strength (Khatri and Kumar, 2009; Tho et al., 2014; Yu et al., 2015). However, plate-type anchors' performance in $\mathrm{MH}$ bearing sediments remains to be experimentally examined.

We investigate the pullout capacity of square anchors shallowly embedded in hydrate-bearing sandy sediments by using small-scale 1-g physical model tests. The pullout tests are conducted on anchors with various widths embedded at different depths. The breakout factor obtained from this study is compared with available numerical and experimental results obtained from conventional soil conditions. The effect of hydrate dissociation on the pullout capacity of the anchors is also examined by conducting anchor pullout tests after the hydrates in the soil experience thermal dissociation. We recognize that it is practically difficult to accurately reproduce field seabed conditions in small 1-g models. However, these models can offer insight into weakening of structures interacting with soils bearing dissociated hydrates, and provide data obtained from well-controlled conditions to calibrate numerical codes for hydrate-related problems (e.g., Kimoto et al., 2010; Rutqvist, 2011). The learned knowledge and the calibrated numerical models can in turn be used for real field conditions. In other words, the objective of the current study is to fulfil the first step in this two-step methodology.

\section{Experimental details}

\subsection{Equipment and model set-up}

As shown in Fig. 1, this study was conducted in a chamber open to the ambient atmosphere. The inside dimensions of the chamber are $1400 \mathrm{~mm} \times 400 \mathrm{~mm} \times 390 \mathrm{~mm} \quad$ (length $\times$ width $\times$ height). The chamber is equipped with a built-in bath cooler that enables the lowest temperature of $-7{ }^{\circ} \mathrm{C}$. A specially-designed loading frame is placed above the chamber in order to provide uplift and lateral forces via an electronic actuator to the model anchors embedded in the soil bed. The model anchors are 5 -mm-thick square steel plates with various plate widths (i.e. $30 \mathrm{~mm}, 45 \mathrm{~mm}$ and $60 \mathrm{~mm}$ ). Each plate is rigidly connected

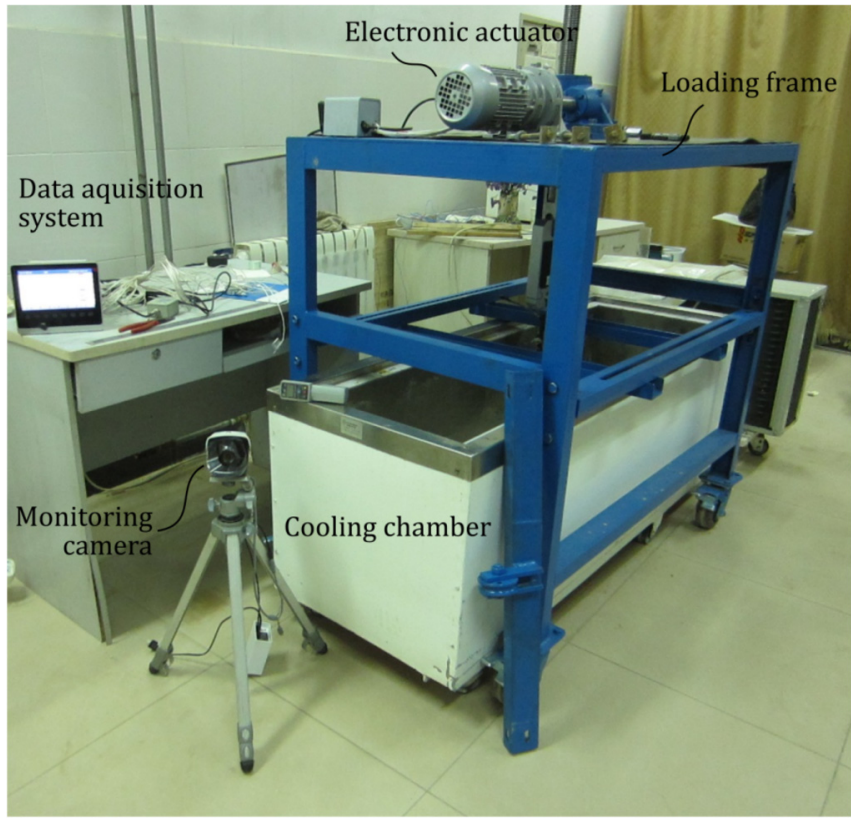

Fig. 1. Apparatus used in the model tests.

to a threaded steel rod $10 \mathrm{~mm}$ in diameter pulled by the actuator through a steel string. The forces applied on the anchors are measured by a load cell, and the displacement of the anchors is measured from the upward movement of the actuator considering the fact that the deformation of steel string is negligible (a few tenth of $\mathrm{mm}$ under the highest load) compared to the total displacement (ranging 8-20 mm) required to mobilize the peak capacity.

The host soil of hydrates used in our study is a silty sand that mimics typical soils from the shallow layer of the South China Sea (Liu et al., 2012). The fine fraction of the sand, i.e. the percent in mass passing No. $200(75 \mu \mathrm{m})$ sieve, is $15.6 \%$. The particle size distribution curve of the sand is presented in Fig. 3, compared with the one obtained from an in situ core sample of hydrate-bearing soils at the South China sea (Liu et al., 2012).

Our laboratory equipment does not allow forming MHs under atmospheric pressure, since the temperature required, below $-80^{\circ} \mathrm{C}$ (Sloan, 1998), is beyond the capacity of our available cooler. As a substitute, we used tetrahydrofuran (THF) hydrates to prepare hydratebearing soil specimens in the chamber. THF hydrates have been adopted as a satisfactory substitute for MHs in many successful physical model tests (Zhang et al., 2015a, b, 2016), owing to their similarity in mechanical and thermal properties (Lee et al., 2007; Yun et al., 2007) to MHs. In addition, a large volume of THF hydrate-bearing sediments can be prepared more uniformly and safely than synthesized MH-bearing sediments, since THF is completely miscible with water in all proportions and forms hydrates under atmospheric pressure at a temperature below $4.4^{\circ} \mathrm{C}$.

We investigated the performance of the anchors under two soil conditions: (1) the hydrate case, in which the soil hosts hydrates in $85 \%$ saturation (i.e., $85 \%$ of the void space filled with hydrates and the remaining void space filled with air in order to eliminate effect of ice); and (2) the hydrate-free case, in which the hydrate-bearing soil has completely dissociated. The hydrate saturation degree (85\%) was selected here to represent the conditions of a highly-concentrated hydrate reservoir possible in the South China Sea, where the hydrate saturation degree ranges up to $90 \%$ (Qian et al., 2017). Pore water in the soil would be problematic, as it could freeze into ice under our test conditions and make it difficult to interpret the effects of hydrates (as well as its dissociation) on anchors' bearing capacity. To avoid this complexity, we picked the THF-to-water mass ratio (19:81) so that all fluid is 
Table 1

A summary of the test conditions.

\begin{tabular}{llllll}
\hline Test & $\begin{array}{l}\text { Soil } \\
\text { conditions }\end{array}$ & Inclination $\alpha\left(^{\circ}\right)$ & $\begin{array}{l}\text { Buried } \\
\text { depth } H \\
(\mathrm{~mm})\end{array}$ & $\begin{array}{l}\text { Plate } \\
\text { width } B \\
(\mathrm{~mm})\end{array}$ & $\begin{array}{l}\text { Buried } \\
\text { depth ratio } \\
H / B\end{array}$ \\
\hline S1-H1 & With hydrates & 0 & 30 & 30 & 1 \\
S1-H2 & With hydrates & 0 & 60 & 30 & 2 \\
S1-H3 & With hydrates & 0 & 90 & 30 & 3 \\
S2-H1 & With hydrates & 0 & 60 & 30 & 2 \\
S2-H2 & With hydrates & 0 & 60 & 45 & 1.33 \\
S2-H3 & With hydrates & 0 & 60 & 60 & 1 \\
S3-H1 & With hydrates & 0 & 60 & 30 & 2 \\
S3-D2 & $\begin{array}{l}\text { Hydrates } \\
\text { dissociated }\end{array}$ & 0 & 60 & 30 & 2 \\
S3-D3 & $\begin{array}{l}\text { Hydrates } \\
\text { dissociated }\end{array}$ & 45 & 60 & 30 & 2 \\
& & & & \\
\hline
\end{tabular}

consumed in forming the hydrates and no free water would be available to form ice.

Table 1 summarizes three series of tests (i.e. S1, S2, S3), each containing three pullout runs in order to take full advantage of the elongated chamber. Series S1 and S2 were conducted on verticallyloaded anchors buried in hydrate-bearing soil for examining the effects of the burial depth and the sizes of anchors. In series S3, the first run was a replicate test of the hydrate-bearing case to check data reliability, whereas the second and third runs were conducted after thermal dissociation of hydrates in order to investigate the performance of the anchors in soil with dissociated hydrates.

Fig. 2 illustrates the model set-up of series S1 as an example. The soil bed is $155 \mathrm{~mm}$ thick, burying three identical 30-mm-wide anchors at depths of 30,60 and $90 \mathrm{~mm}$, respectively. The model dimensions are chosen in such a way that (1) the chamber boundaries do not impact the

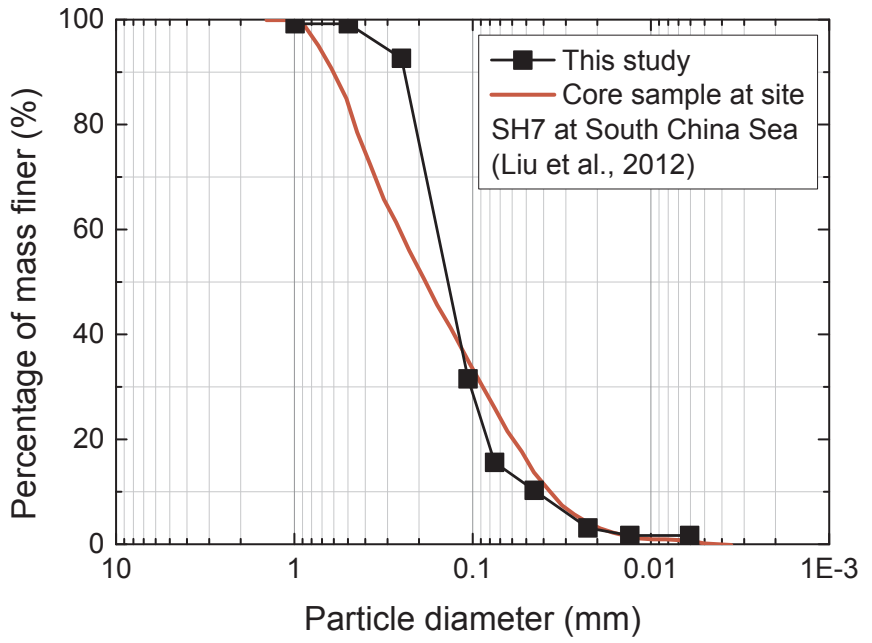

Fig. 3. Particle size distribution curve of the host silty sand.

breakout cones mobilized in the soil failure mechanism, and (2) the cones do not interfere with each other. The set-ups of series S2 and S3 are similar, except that a plate heater is buried at $50 \mathrm{~mm}$ below the second and third anchors in series S3 to facilitate thermal dissociation.

\subsection{Testing procedure}

Each test is performed in four steps: specimen preparation, THF solution percolation, hydrate formation (and dissociation in series S3), and pullout loading. First, the pulverized sand is mixed with distilled water to reach a moisture content of $7 \%$, and then compacted in four

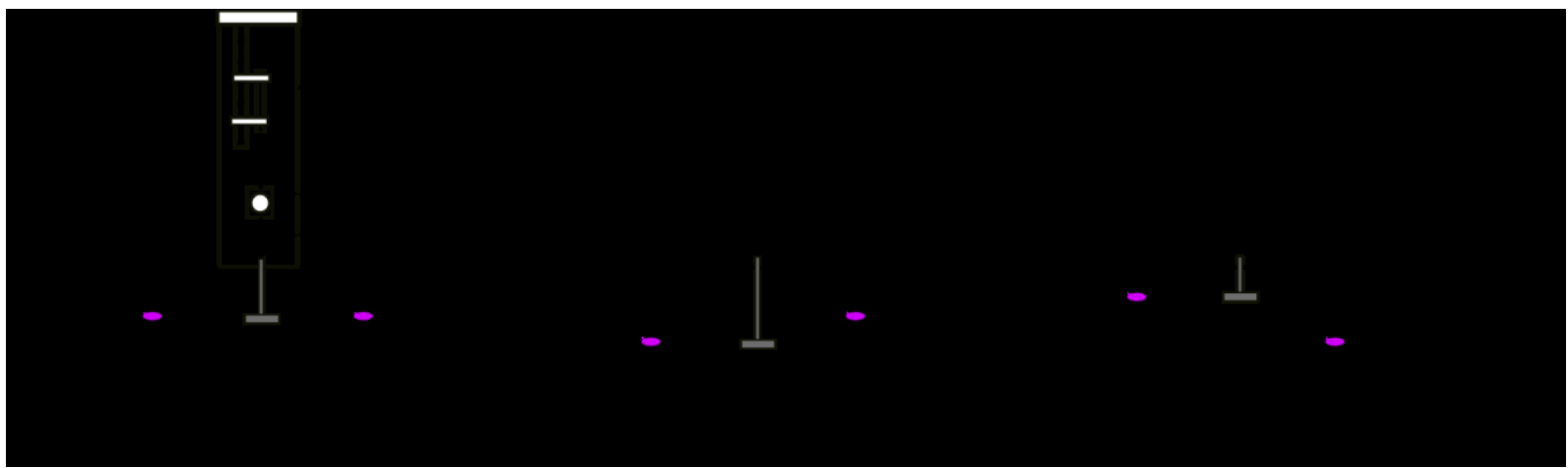

(a) Side view

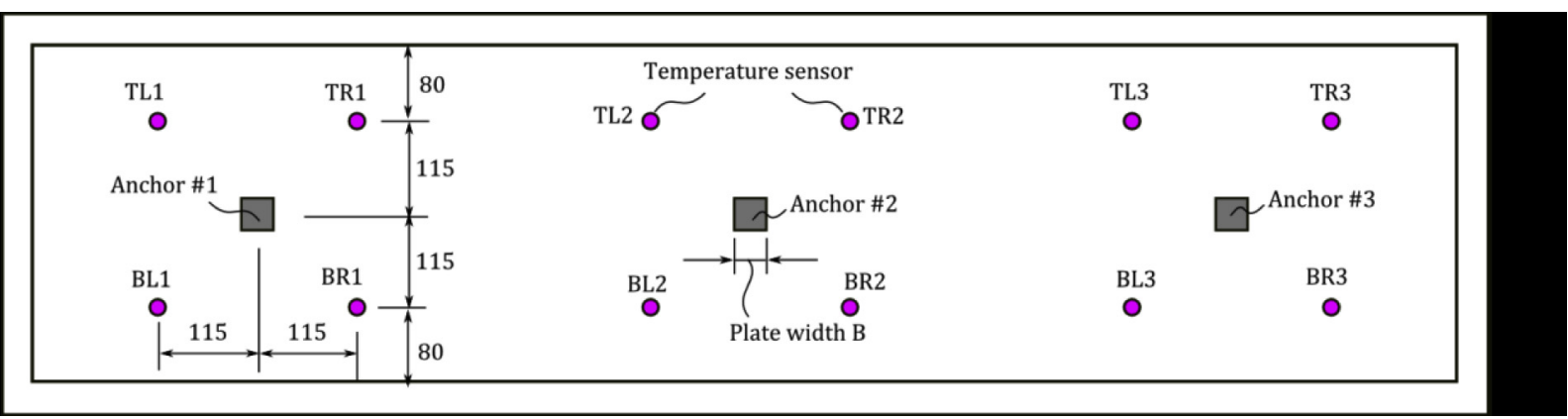

(b) Top view

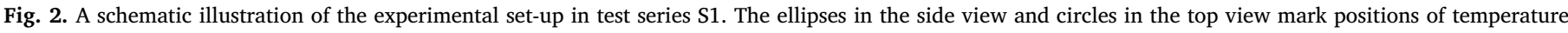
sensors (marked as TL1 to 3 , TR1 to 3 , BL1 to 3 , and BR1 to 3). All dimensions are in mm. 
layers in the chamber to reach a target dry density of $1.60 \mathrm{~g} / \mathrm{cm}^{3}$. The plate anchors and sensors are buried at intended positions denoted in Fig. 2. Second, THF and distilled water are mixed in a selected mass ratio to allow THF and water to completely turn into THF hydrates. According to the target hydrate saturation $(85 \%)$ and the total pore volume in the soil specimen, a desired volume (approximately 15 litters) of a mixture of THF and distilled water are prepared and sprayed slowly on the soil bed by 10 partitions in $10 \mathrm{~h}$. Third, the chamber is cooled down to enable hydrate formation, and is blanketed by a plastic cover to avoid evaporation of THF and water. The temperature in the soil bed is maintained at $-7^{\circ} \mathrm{C}$ for $3-4$ days to ensure that hydrates are completely formed in the entire soil bed. Fourth, the buried plate anchors are loaded with vertical upwards forces at a constant displacement rate of $0.365 \mathrm{~mm} / \mathrm{min}$ to achieve a quasi-static loading condition (i.e. no significant inertial effects) (Delhomme et al., 2016). In series S3, after the first anchor is pulled out, the cooler is turned off and the buried heater is turned on to dissociate the hydrates. After complete dissociation, the remaining two anchors are successively pulled out via a vertical load and an inclined load at $45^{\circ}$ to the vertical, respectively.

It is practically difficult to generate a large volume of hydratebearing soil specimen with uniform properties. Besides the spraying procedure adopted, we had explored other alternatives including seeping THF solution via pressurized air from the base of the specimen, and mixing THF solution with the host soil before compaction. However, the former suffered from equipment failures, and the latter encountered troubles as we compacted a silty sand with very high moisture content. We checked the homogeneity of the specimens resulting from the praying procedure. In the end of every test series, the remaining soil in the chamber is dissociated at room temperature, and soil samples were collected at different locations and depths to measure the moisture content and dry density. The values of the back-calculated hydrate saturation had a mean of $82.1 \%$ and a standard deviation of $4.1 \%$ for the samples near the base of the chamber. The samples from shallower depths tended to have slightly lower saturation due to water and THF evaporation. This indicates that hydrates were able to form approximately at the target hydrate saturation throughout the entire depth of the experiment chamber.

The temperature in the soil bed was monitored during every test series. As an example, Fig. 4 presents the temperature readings during series $\mathrm{S} 1$. The temperature does not vary significantly at different locations except during the phase of hydrate formation (41-112 $\mathrm{h}$ in Fig. 4). As shown in the inset in Fig. 4, when the temperature drops to

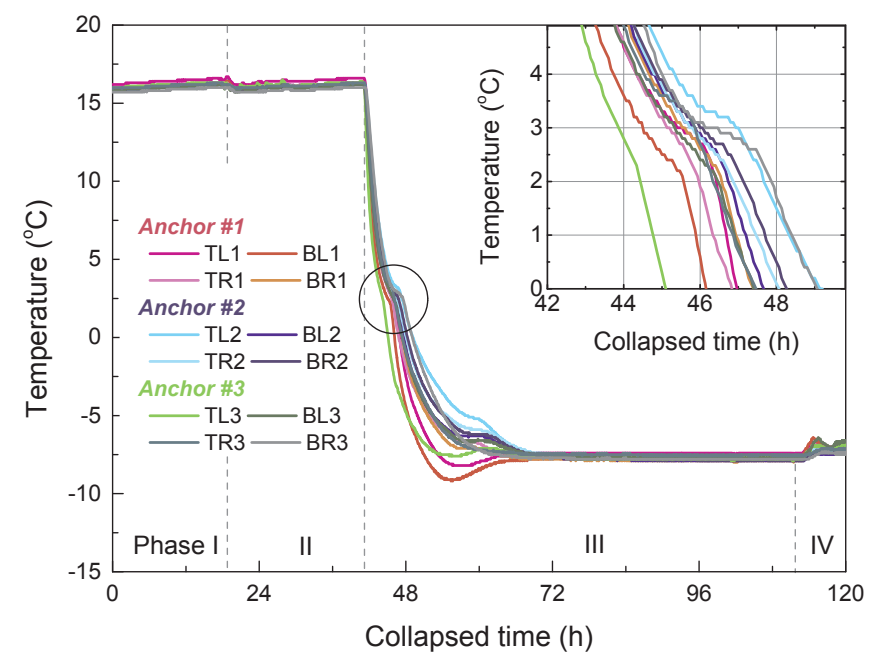

Fig. 4. Variation of temperature with time during the entire process of series S1. The positions of temperature sensors are given in Fig. 2. Phase I: specimen preparation, Phase II: THF solution percolation, Phase III: hydrate formation, Phase IV: pullout loading. the equilibrium temperature of THF hydrates (at about $4{ }^{\circ} \mathrm{C}$ ), the drop slows down. This indicates that hydrates formation, which is an exothermic process, starts. Nevertheless, the temperature ultimately stays at around $-7^{\circ} \mathrm{C}$.

\subsection{Soil characteristics}

To characterize strengths of the soil in the model tests, we conducted triaxial compression tests on hydrate-bearing specimens before and after hydrate dissociation. The model tests were likely under a partially-drained condition, because under a high hydrate saturation (85\%) the pores in the soil could be sealed by hydrates and may not interconnect everywhere in such a large volume of soil specimen in the model tests. To avoid uncertainty in a partially-drained system, we performed the triaxial tests under undrained conditions, considering that the strength do not significantly deviate from that under a partially-drained condition for unsaturated soil samples. For comparison, a set of triaxial compression tests were also conducted on specimens with $85 \%$ void volume filled with THF-water solution (THF-water mass ratio $19: 81$ ), termed "wet specimens". The void ratio is 0.68 in all specimens. The confining pressures ranged from $50 \mathrm{kPa}$ to $400 \mathrm{kPa}$ to cover the stress range of our model tests. Table 2 summarizes the properties of the host soil and the hydrate-bearing specimens. Fig. 5a to c presents the stress-strain curves obtained from different specimens, and Fig. $5 \mathrm{~d}$ plots the derived Mohr's circles at failure and the corresponding strength envelopes. The hydrate-bearing specimens before dissociation exhibit strain-softening (Fig. 5a) because of particle debonding under loading (Cuccovillo and Coop, 1997). The corresponding Mohr-Coulomb's strength envelope (Fig. 5d) is above and nearly parallel to the one obtained from the wet specimens. This indicates that the presence of hydrates significantly enhances the inter-particle cementation, so therefore increases the apparent cohesion of the specimen (i.e., the intersect of the strength envelope on the shear stress axis). However, the presence of hydrates has marginal effect on the internal friction angle. This is in good agreement with results in Masui et al. (2005). As shown in Fig. 5b, the shear strength in the specimens significantly drops after hydrates dissociation, leading to a strength envelope even far below the one obtained from the wet specimens (Fig. 5d). Two mechanisms contribute to the dissociation-induced strength reduction. Besides the loss of hydrate cementation, fabric alteration (i.e., rearrangement of soil grains) during the forming and dissociation of hydrate also impacts the shear strength of the specimens. As suggested in Lee et al. (2010), hydrate-bearing soils tend to expand (at a high hydrate saturation) in the forming of hydrate and contract during hydrate's dissociation. Consequently, soil grains are moved apart as hydrates form and expand in volume; as hydrates dissolve, the soil skeleton is destabilized due to the expulsion of hydrates that previously constitute the skeletal frame together with soil grains.

Table 2

Properties of the host sand and the hydrate-bearing specimen.

\begin{tabular}{ll}
\hline Parameter & Value \\
\hline Host sand (soil skeleton) & \\
Average particle size & $0.16 \mathrm{~mm}$ \\
Specific gravity & 2.67 \\
Dry density & $1.60 \mathrm{~g} / \mathrm{cm}^{3}$ \\
Void ratio & 0.68 \\
Relative density & $54 \%$ \\
Hydrate-bearing specimen & \\
Hydrate density & $0.997 \mathrm{~g} / \mathrm{cm}^{3}$ \\
Hydrate saturation & $85 \%$ \\
Water saturation & $0 \%$ \\
Cohesion & $510 \mathrm{kPa}$ (before diss.), $3 \mathrm{kPa}$ (after diss.) \\
Internal friction angle & $17^{\circ}$ (before diss.), $0.8^{\circ}$ (after diss.)
\end{tabular}




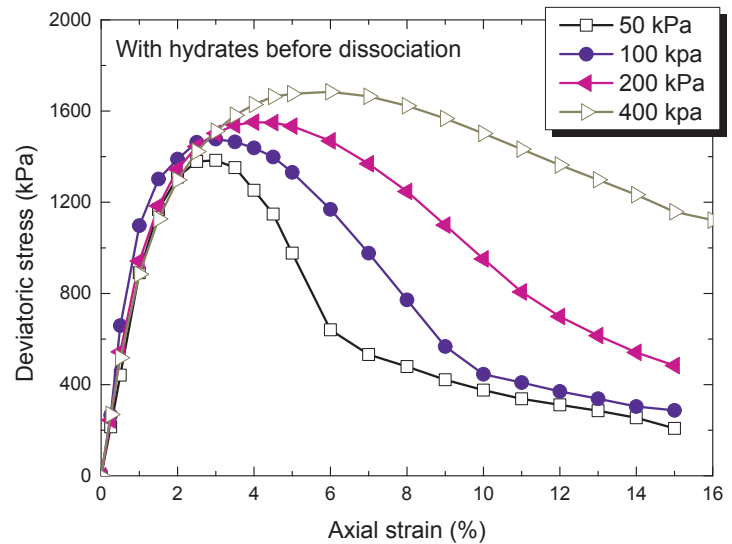

(a)

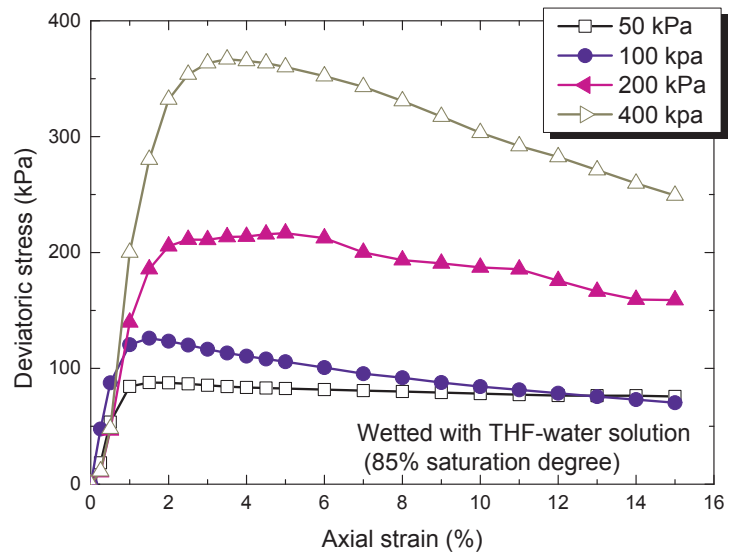

(c)

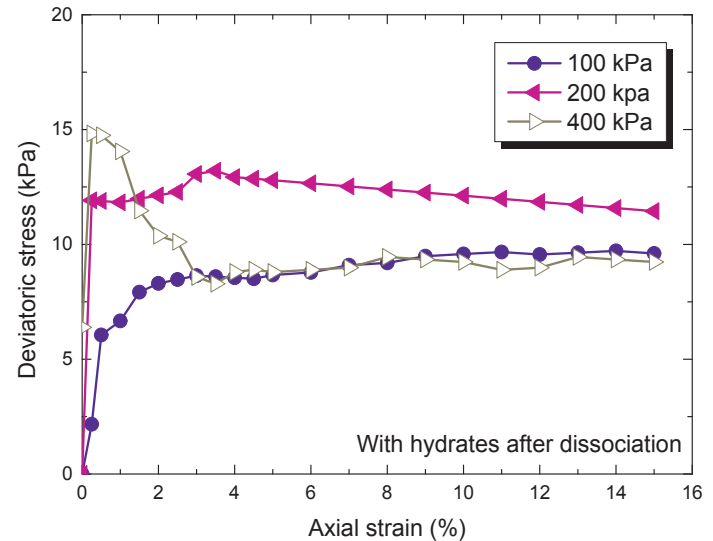

(b)

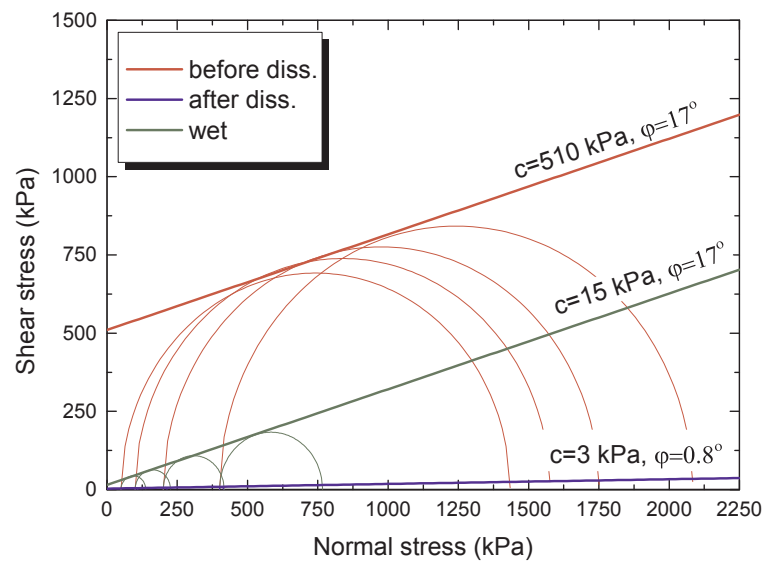

(d)

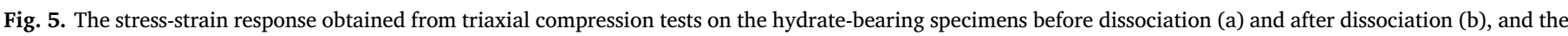

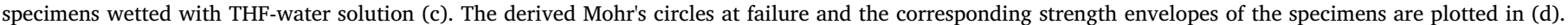

\section{Results}

\subsection{Load-displacement response and pullout capacity}

Fig. 6 presents the load against the displacement normalized by the buried depth of the anchors obtained from series S2. The net pullout force (excluding the weight of the anchor) increases up to a peak (defined herein as the anchor pullout capacity), and then abruptly drops. At a constant burial depth, the pullout capacity increases with the increase of the anchor width as expected, whereas the vertical displacement required for mobilizing the capacity decreases. Table 3 summarizes the values of the pullout capacity obtained from all tests. As we have expected, the pullout capacity increases with the increase of the buried depth for a fixed plate width. We also noted that test S2-H1 resulted in significantly lower pullout capacity than S1-H2 and S3-H1, although they had the same test conditions. This difference is believed to be caused by the heterogeneity of the soil bed and the variation in time spent in hydrate formation. Series S2 was performed before S1 and S3. Series S2 took four days to form hydrates and fine fissures appeared on the surface of the soil bed before loading. To reduce ground fissures, hydrate formation was shortened by $24 \mathrm{~h}$ in the successive series $\mathrm{S} 1$ and S3. This change causes higher values of the pullout capacity in series S1 and S3, and nevertheless it does not significantly change the general trend of the load-displacement curves.

Here we present the results in a manner less dependent on the model scale by using a dimensionless descriptor, the breakout factor $N_{c}$,

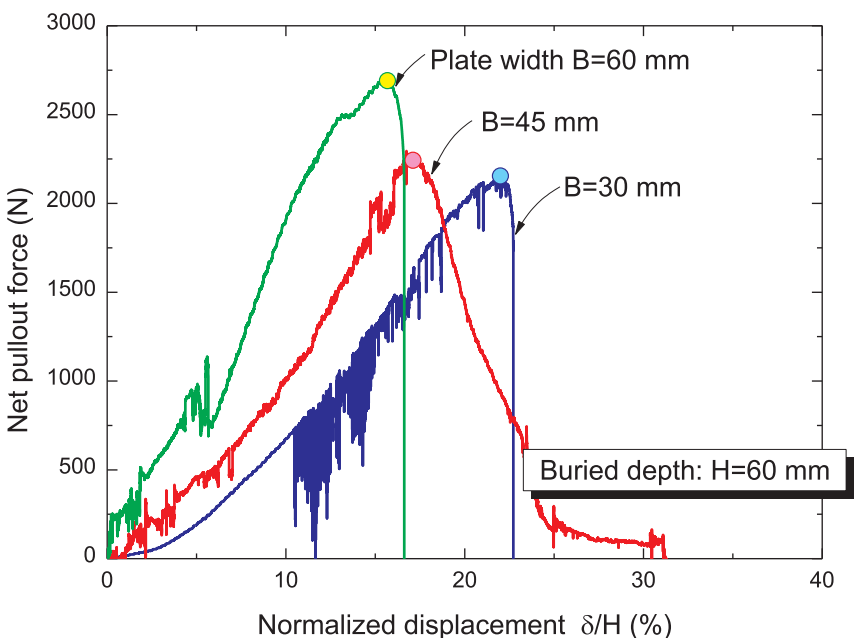

Fig. 6. Load-displacement diagrams under various plate widths. The ultimate pullout capacity is defined as the peaks and marked with circles.

defined for anchors buried in clays under undrained conditions (Merifield et al., 2001; Rowe and Davis, 1982). This factor can be computed as: 
Table 3

A summary of the pullout test results.

\begin{tabular}{lll}
\hline Test & Pullout capacity $Q_{\mathrm{u}}(\mathrm{N})$ & Breakout factor $N_{c o}$ \\
\hline S1-H1 & 1125 & 2.45 \\
S1-H2 & 3636 & 7.92 \\
S1-H3 & 4692 & 10.22 \\
S2-H1 & 2168 & 4.72 \\
S2-H2 & 2295 & 2.22 \\
S2-H3 & 2693 & 1.46 \\
S3-H1 & 3287 & 7.16 \\
\hline
\end{tabular}

$N_{c}=\frac{Q_{u}}{A c_{u}}=\frac{Q_{u}-A H \gamma}{A c_{u}}+\frac{\gamma H}{c_{u}}=N_{c 0}+\frac{\gamma H}{c_{u}}$

where $N_{c o}$ is the breakout factor under conditions of weightless soil (denoted as the net breakout factor for short), and $Q_{u}$ is the pullout capacity of the anchor with an area $A$ buried at depth $H$ in a clay with an undrained shear strength $c_{u}$ and a unit weight of $\gamma$.

As confirmed in traxial compression tests, the hydrate-bearing soil in our study has a high apparent cohesion due to hydrate cementation between soil grains. The contribution of this cohesion to shear strength of the hydrate-bearing soil is significant especially at low confining pressure when the contribution from the internal friction is small. This "cohesion-dominated" strength behaviour in the hydrate-bearing soil is similar to that of clays under undrained conditions, although the origins of the strength in these two types of soils are different. Through an analogy between these two soil types, we replace $c_{u}$ in Eq. (1) with the apparent cohesion $c$ of the hydrate-bearing soil and calculated $N_{c o}$ from the test data according to Eq. (1). Fig. 7 plots the resulting $N_{c o}$ against the embedment ratio $(H / B)$. Note that the net breakout factor is used here for comparison in order to reduce the effect of different weights used in various studies. The published data for square anchors buried in undrained clays with $c_{u}$ ranging $225-380 \mathrm{kPa}$ (Das, 1978) are also superimposed in Fig. 7 for comparison. As shown in this figure, $N_{c o}$ obtained from our model tests correlates well with the embedment ratio, and in general follows the trend formed by the data obtained from clays under undrained conditions (Das, 1978). This indicates that the hydrate-bearing soil interacts with the plate anchor in a similar manner as clays under undrained conditions, where the apparent cohesion resulting from cementation dominantly affects the anchor capacity. We noted that some of our data points obtained at larger embedment ratios (e.g., $H / B=2$ and 3 ) are higher than the experimental results by Das (1978). Despite the lack of replicate tests due to constraints from

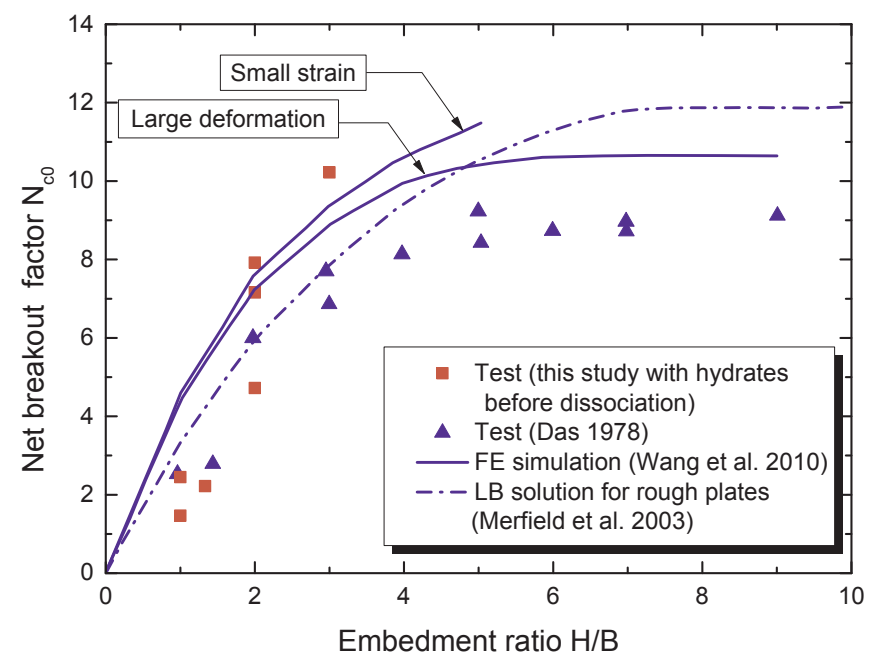

Fig. 7. The net breakout factor varying against the embedment ratio in comparison with published testing data (Das, 1978) and published simulations (Merfield et al., 2003; Wang et al., 2010).

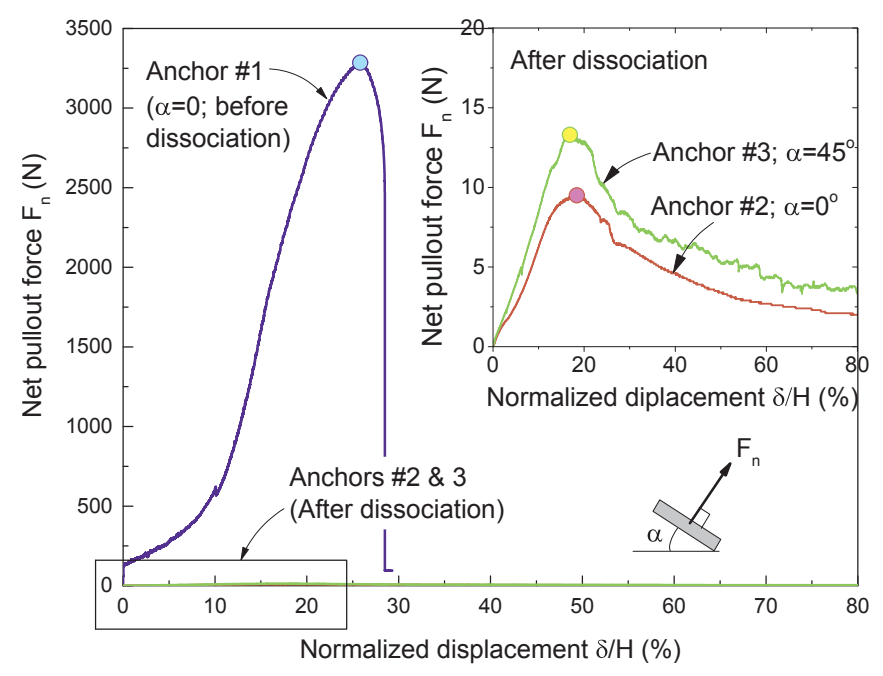

Fig. 8. Load-displacement diagrams of plate anchors embedded in the hydratebearing ground and dissociated ground (the inset).

laboratory availability, this behaviour is believed to be consistent with expected hydrate-bearing soil properties: the pressure-dependent frictional strength of hydrate-bearing soil increasingly contributes to the anchor capacity as the anchor embedment increases. Within the range of the embedment ratio tested in this study, $N_{c o}$ does not reach a steady value, indicating that all the anchors in this study are in the "shallow" category, for which the pullout capacity is sensitive to the buried depth. Fig. 7 also compares our experimental results with the published numerical analyses for rectangular plate anchors shallowly embedded in homogenous clays under undrained conditions using different approaches, including the plasticity lower bound solution (Merifield et al., 2003), the conventional small-strain (based on un-deformed original geometry) and large-deformation (tracking evolution of soil geometry) finite element (FE) simulations (Wang et al., 2010). The small-strain FE curve fits our data the best particularly at large embedment depths.

\subsection{Effect of hydrate dissociation}

Fig. 8 plots the load-displacement curves obtained before and after hydrate dissociation (series S3). As the hydrates completely dissociate, the load-displacement response exhibits ductile feature; the load gradually declines when it passes the peak. For the vertically loaded anchor (i.e. $\alpha=0^{\circ}, \alpha$ is the loading angle to the vertical as denoted in Fig. 8), the pullout capacity declines to $9.5 \mathrm{~N}$ (i.e, about $0.3 \%$ of the original value) after hydrate dissociation. This dramatic reduction in capacity occurs due to a significant decrease in shear strength of the soil associated with hydrate dissociation. The hydrate saturation in the soil is rather high ( $85 \%)$ so a significant portion of the hydrates likely act as inter-particle cementations (Waite et al., 2009), resulting in a high apparent cohesion of the soil. This has been confirmed by a high value (i.e., $510 \mathrm{kPa}$ ) of cohesion obtained from triaxial compression tests on the hydrate-bearing specimens (see Section 2.3). As the hydrates dissociate under the temperature rise, inter-particles cementations disappear, causing a significant loss of shear strength in the soil and in turn a great drop in the bearing capacity of the anchors. The pullout capacity of the anchor under the inclined load is larger than that of verticallyloaded anchor. This agrees with the existing studies on the effect of inclined loading (Murray and Geddes, 1989).

\subsection{Failure modes}

Fig. 9 presents the failures modes of different cases. The breakout cones from the tests of the hydrate-bearing case are similar in shape, and Fig. 9a and b presents the cone obtained from a typical test (S2-H1: 


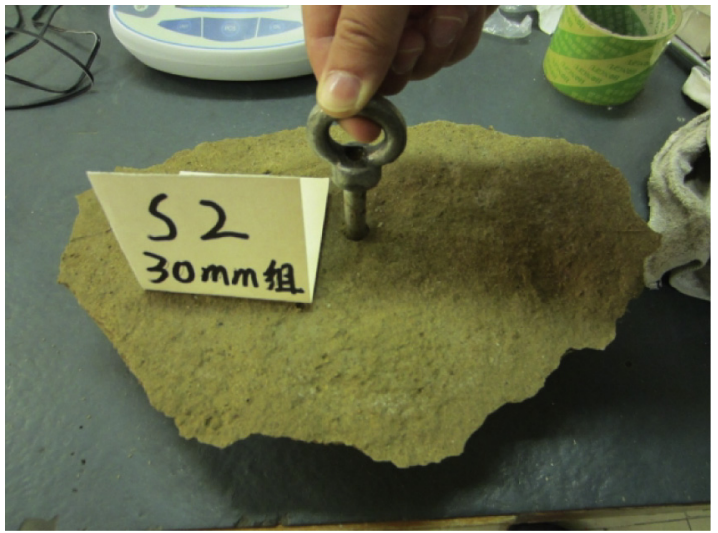

(a)

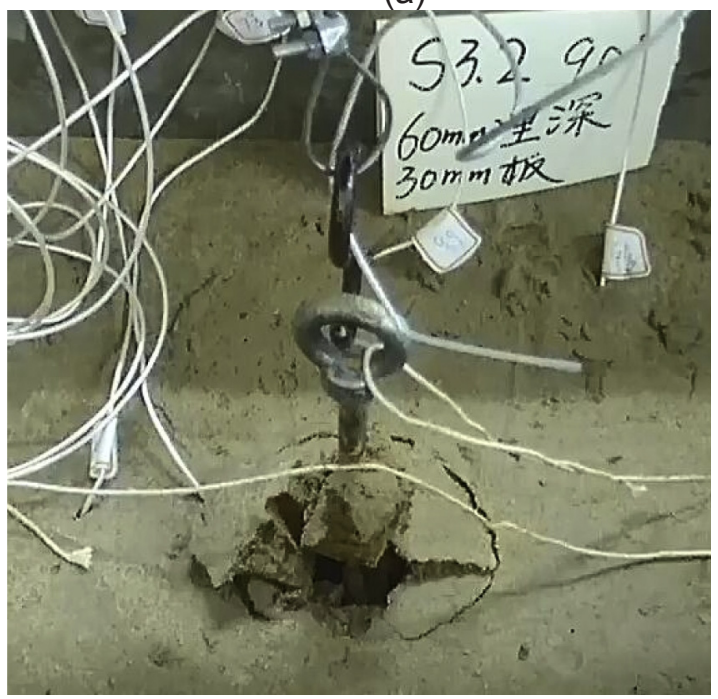

(c)

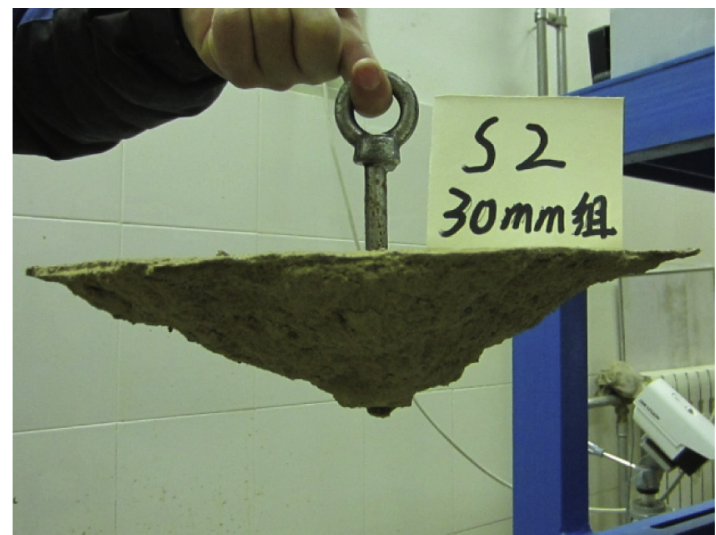

(b)

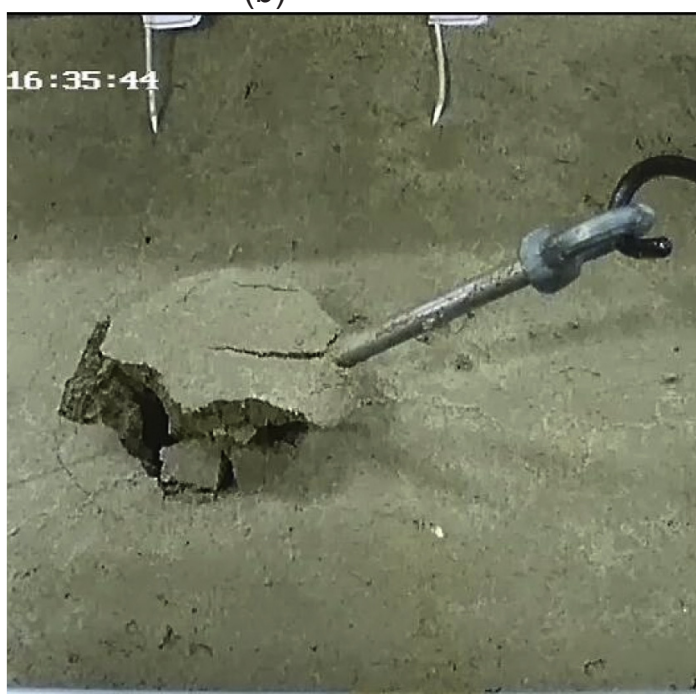

(d)

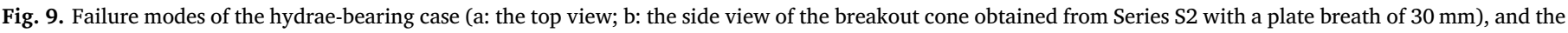
hydrate-dissociated case (c: vertically-loaded anchor; d: obliquely-loaded anchor, embedded at a depth of $60 \mathrm{~mm}$ with a plate width of $30 \mathrm{~mm}$ ).

$B=30 \mathrm{~mm}, H=60 \mathrm{~mm}$ ) of this case. The side inclination of the cones varies in a range of $58^{\circ}$ to $61^{\circ}$ to the vertical depending on the plate width and buried depth. During loading, breakout occurs suddenly with little manifestation on the ground surface. These observations are consistent with the behaviour of anchorages in strongly-cemented materials such as concrete (Delhomme et al., 2015). Fig. 9c and d presents the breakout pattern under dissociated conditions for the vertically- and obliquely-loaded anchors, respectively. The projected area of the failure cone on the ground surface is much smaller than that in the hydrate-bearing case. As the load increases, the breakout proceeds gradually with accumulating noticeable cracks on the ground surface.

\section{Conclusions}

Model tests were conducted to investigate the pullout capacity of square anchors shallowly buried in hydrate-bearing soil. Under uplift loads, the anchor is pulled out in a brittle manner forming a truncated cone in hydrate-bearing ground. Under the test conditions with the embedment ratio less than 3 , the anchors exhibit "shallow anchor behaviour" which is characterized by a pullout capacity dependent on the buried depth of the anchors. The ultimate pullout capacity of the anchors can be estimated through a dimensionless breakout factor, which increases with higher embedment ratio following a similar trend for shallow plate anchors buried in clays under undrained conditions. When hydrates dissociate, the pullout capacity of the anchor drops to
$0.3 \%$ of the initial value. This pronounced reduction highlights the need for further investigation on the effect of oceanic hydrates dissociation due to natural or anthropologic perturbation on engineering performance of offshore facilities.

\section{Acknowledgement}

We are grateful to anonymous reviewers for their valuable comments and suggestions. This study is jointly funded by National Nature Science Foundation of China (with grant No. 41572267, 51639008, 51239010), and the Fundamental Research Funds for the Central Universities. The authors would like to appreciate Dr. Pengcheng Fu at the Lawrence Livermore National Laboratory, Prof. Xiaobing Lu at the Institute of Mechanics, Chinese Academy of Sciences, and Prof. Xiangdong $\mathrm{Hu}$ at Tongji University for providing constructive discussion. Associate Researcher Jing Zhao and graduate student Dashuang Luo at the Institute of Mechanics, Chinese Academy of Sciences assisted the experiments.

\section{References}

Ardebili, Z.A., Gabr, M.A., Rahman, M.S., 2016. Uplift capacity of plate anchors in saturated clays: analyses with different constitutive models. Int. J. GeoMech. 16, 4015053. https://doi.org/10.1061/(ASCE)GM.1943-5622.0000518.

Bradshaw, A.S., Giampa, J.R., Gerkus, H., Jalilvand, S., Fanning, J., Nanda, S., Gilbert, R., Gavin, K., Sivakumar, V., 2017. Scaling considerations for 1-g model horizontal plate anchor tests in sand. Geotech. Test J. 39, 1006-1014. https://doi.org/10.1520/ GTJ20160042. 
Chow, S.H., O'Loughlin, C.D., Corti, R., Gaudin, C., Diambra, A., 2015. Drained cyclic capacity of plate anchors in dense sand: experimental and theoretical observations. Géotech. Lett. 5, 80-85. https://doi.org/10.1680/geolett.15.00019.

Coetzee, C.J., Vermeer, P.A., Basson, A.H., 2005. The modelling of anchors using the material point method. Int. J. Numer. Anal. Methods GeoMech. 29, 879-895. https:// doi.org/10.1002/nag.439.

Cuccovillo, T., Coop, M.R., 1997. Yielding and pre-failure deformation of structured sands. Geotechnique 47 (3), 491-508.

Das, B.M., 1995. Behavior of a shallow plate anchor in clay under sustained loading. Mar. Georesour. Geotechnol. 13, 417-428. https://doi.org/10.1080/ 10641199509388296

Das, B.M., 1978. Model tests for uplift capacity of foundations in clay. Japan. Soc. Soil Mech. Found. Eng. 18, 17-24. https://doi.org/10.3208/sandf1972.18.2 17.

Delhomme, F., Roure, T., Arrieta, B., Limam, A., 2016. Pullout behavior of cast-in-place headed and bonded anchors with different embedment depths. Mater. Struct. 49, 1843-1859. https://doi.org/10.1016/j.nucengdes.2015.03.012.

Delhomme, F., Roure, T., Arrieta, B., Limam, A., 2015. Static and cyclic pullout behavior of cast-in-place headed and bonded anchors with large embedment depths in cracked concrete. Nucl. Eng. Des. 287, 139-150. https://doi.org/10.1016/j.nucengdes.2015. 03.012.

Ilamparuthi, K., Dickin, E.A., Muthukrisnaiah, K., 2002. Experimental investigation of the uplift behaviour of circular plate anchors embedded in sand. Can. Geotech. J. 39, 648-664. https://doi.org/10.1139/T02-005.

Ilamparuthi, K., Muthukrishnaiah, K., 1999. Anchors in sand bed: delineation of rupture surface. Ocean Eng. 26, 1249-1273.

Khatri, V.N., Kumar, J., 2009. Vertical uplift resistance of circular plate anchors in clays under undrained condition. Comput. Geotech. 36, 1352-1359. https://doi.org/10. 1016/j.compgeo.2009.06.008.

Kimoto, S., Oka, F., Fushita, T., 2010. A chemo-thermo-mechanically coupled analysis of ground deformation induced by gas hydrate dissociation. Int. J. Mech. Sci. 52 365-376. https://doi.org/10.1016/j.ijmecsci.2009.10.008.

Kvenvolden, K. a, Lorenson, T.D., 2001. The global occurrence of natural gas hydrate. Nat. Gas Hydrates Occur. Distrib. Detect. 3-18. https://doi.org/10.1029/ GM124p0003.

Kwon, T.H., Cho, G.C., Santamarina, J.C., 2008. Gas hydrate dissociation in sediments: pressure-temperature evolution. Geochem. Geophys. Geosyst. 9, Q03019. https://doi. org /10.1029/2007GC001920.

Kwon, T.H., Oh, T.M., Choo, Y.W., Lee, C., Lee, K.R., Cho, G.C., 2013. Geomechanical and thermal responses of hydrate-bearing sediments subjected to thermal stimulation: physical modeling using a geotechnical centrifuge. Energy Fuels 27 (8), 4507-4522.

Kwon, T.H., Song, K.I., Cho, G.C., 2010. Destabilization of marine gas hydrate-bearing sediments induced by a hot wellbore: a numerical approach. Energy Fuels 24, 5493-5507. https://doi.org/10.1021/ef100596x.

Lee, J.Y., Yun, T.S., Santamarina, J.C., Ruppel, C., 2007. Observations related to tetrahydrofuran and methane hydrates for laboratory studies of hydrate-bearing sediments. Geochem. Geophys. Geosyst. 8. https://doi.org/10.1029/2006GC001531.

Lee, J.Y., Santamarina, J.C., Ruppel, C., 2010. Volume change associated with formation and dissociation of hydrate in sediment. Geochem. Geophys. Geosyst. 11. https:// doi.org/10.1029/2009GC002667.

Liu, C., Ye, Y., Meng, Q., He, X., Lu, H., Zhang, J., Liu, J., Yang, S., 2012. The characteristics of gas hydrates recovered from shenhu area in the South China sea. Mar. Geol. 307-310, 22-27. https://doi.org/10.1016/j.margeo.2012.03.004.

Masui, A., Haneda, H., Ogata, Y., Aoki, K., 2005. Effects of methane hydrate formation on shear strength of synthetic methane hydrate sediments. Fifteenth Int. Offshore Polar Eng. Conf. 8, 364-369. https://doi.org/10.1063/1.1804617.

Merifield, R.S., Lyamin, A.V., Sloan, S.W., 2006. Three-dimensional lower-bound solutions for the stability of plate anchors in sand. Geotechnique 56, 123-132.

Merifield, R.S., Lyamin, A.V., Sloan, S.W., Yu, H.S., 2003. Three-dimensional lower bound solutions for the stability of plate anchors in Clay. J. Geotech. Geoenviron. Eng. 129, 243-253.

Merifield, R.S., Yu, H.S., Sloan, S.W., 2001. Stability of plate anchors in undrained clay. Geotechnique 51, 141-153. https://doi.org/10.1680/geot.2001.51.2.141.

Moridis, G.J., Berkeley, L., Collett, T.S., Survey, U.S.G., 2011. Challenges, uncertainties and issues facing gas production from gas hydrate deposits. In: The Proceedings of SPE Unconventional Gas Conference. Pittsburgh, Pennsylvania, USA, pp. 76-112. https://doi.org/10.2118/131792-PA.

Murray, E.J., Geddes, J.D., 1989. Resistance of passive inclined anchors. Geotechnique 39, 417-431.

Qian, J., Wang, X., Collett, T.S., Dong, D., Guo, Y., Su, P., Liang, J., 2017. Gas hydrate accumulation and saturations estimated from effective medium theory in the eastern Pearl River Mouth Basin, South China Sea. Interpretation 5 (3), SM33-SM48. https:// doi.org/10.1190/INT-2016-0217.1.

Randolph, M.F., Gaudin, C., Gourvenec, S.M., White, D.J., Boylan, N., Cassidy, M.J., 2011. Recent advances in offshore geotechnics for deep water oil and gas developments. Ocean Eng. 38, 818-834. https://doi.org/10.1016/j.oceaneng.2010.10.021.

Rao, K.S.S., Kumar, J., 1994. Vertical uplift capacity of horizontal anchors. J. Geotech. Eng. 120, 1134-1147. (ASCE)0733-9410(1994)120:7(1134). https://doi.org/10. $1061 /$.

Rowe, R.K., Davis, E.H., 1982. Behaviour of anchor plates in sand. Geotechnique 32, 25-41. https://doi.org/10.1680/geot.1982.32.1.9.

Rutqvist, J., 2011. Status of the TOUGH-FLAC simulator and recent applications related to coupled fluid flow and crustal deformations. Comput. Geosci. 37, 739-750. https:// doi.org/10.1016/j.cageo.2010.08.006.

Sloan, E.D., 1998. Clathrate Hydrates of Natural Gas, second ed. Marcel Dekker, Inc, New York.

Tho, K.K., Chen, Z., Leung, C.F., Chow, Y.K., 2014. Pullout behaviour of plate anchor in clay with linearly increasing strength. Can. Geotech. J. 51, 92-102.

Thorne, C.P., Wang, C.X., Carter, J.P., 2004. Uplift capacity of rapidly loaded strip anchors in uniform strength clay. Geotechnique 54, 507-517. https://doi.org/10.1680/ geot.2004.54.8.507.

Wang, D., Gaudin, C., Randolph, M.F., 2013. Large deformation finite element analysis investigating the performance of anchor keying flap. Ocean Eng. 59, 107-116. https://doi.org/10.1016/j.oceaneng.2012.12.010.

Waite, W.F., Santamarina, J.C., Cortes, D.D., Dugan, B., Espinoza, D.N., Germaine, J., Jang, J., Jung, J.W., Kneafsey, T.J., Shin, H., Soga, K., Winters, W.J., Yun, T.S., 2009. Physical properties of hydrate-bearing sediments. Rev. Geophys. 47, 1-38. https:// doi.org/10.1029/2008RG000279.

Wang, D., Hu, Y., Randolph, M.F., 2010. Three-dimensional large deformation finiteelement analysis of plate anchors in uniform clay. J. Geotech. Geoenviron. Eng. 136, 355-365. https://doi.org/10.1061/(ASCE)GT.1943-5606.0000210.

Yu, S.B., Hambleton, J.P., Sloan, S.W., 2015. Undrained uplift capacity of deeply embedded strip anchors in non-uniform soil. Comput. Geotech. 70, 41-49. https://doi. org/10.1016/j.compgeo.2015.07.014.

Yun, T.S., Santamarina, C.J., Ruppel, C., 2007. Mechanical properties of sand, silt, and clay containing tetrahydrofuran hydrate. J. Geophys. Res. Solid Earth 112, B04106. https://doi.org/10.1029/2006JB004484.

Zhang, J.H., Lin, H.L., Wang, K.Z., 2015a. Centrifuge modeling and analysis of submarine landslides triggered by elevated pore pressure. Ocean Eng. 109, 419-429. https://doi. org /10.1016/j.oceaneng.2015.09.020.

Zhang, X.H., Lu, X.B., Chen, X.D., Zhang, L.M., Shi, Y.H., 2016. Mechanism of soil stratum instability induced by hydrate dissociation. Ocean Eng. 122, 74-83. https://doi.org/ 10.1016/j.oceaneng.2016.06.015.

Zhang, X.H., Lu, X.B., Shi, Y.H., Xia, Z., Liu, W.T., 2015b. Centrifuge experimental study on instability of seabed stratum caused by gas hydrate dissociation. Ocean Eng. 105, 1-9. https://doi.org/10.1016/j.oceaneng.2015.06.006. 